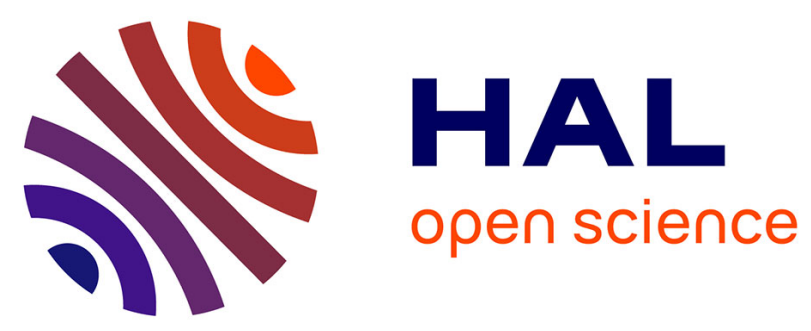

\title{
D'un service transversal prestataire de services à une UFR décisionnaire de la politique des langues : exemple concret de la structuration d'une UFR Lansad à l'université de Lorraine et de ses impacts en termes de recherche et formations
}

Nicolas Molle, Justine Paris, Carine Martin

\section{To cite this version:}

Nicolas Molle, Justine Paris, Carine Martin. D'un service transversal prestataire de services à une UFR décisionnaire de la politique des langues: exemple concret de la structuration d'une UFR Lansad à l'université de Lorraine et de ses impacts en termes de recherche et formations. Recherche et pratiques pédagogiques en langues de spécialité - Cahiers de l'APLIUT, 2019, Le secteur Lansad, fédérateur de diversité(s) ?, 38 (1), 10.4000/apliut.6653 . hal-02367644

\section{HAL Id: hal-02367644 \\ https://hal.science/hal-02367644}

Submitted on 2 Dec 2019

HAL is a multi-disciplinary open access archive for the deposit and dissemination of scientific research documents, whether they are published or not. The documents may come from teaching and research institutions in France or abroad, or from public or private research centers.
L'archive ouverte pluridisciplinaire HAL, est destinée au dépôt et à la diffusion de documents scientifiques de niveau recherche, publiés ou non, émanant des établissements d'enseignement et de recherche français ou étrangers, des laboratoires publics ou privés. 
Cahiers de l'Apliut

D’un service transversal prestataire de services à une UFR décisionnaire de la politique des langues : exemple concret de la structuration d'une UFR Lansad à l'université de Lorraine et de ses impacts en termes de recherche et formations

From a Cross-Department Service Provider to a Policymaker for Languages: The Example of the UFR Lansad at the University of Lorraine and its Impact in Research and Student Training

Nicolas Molle, Justine Paris et Carine Martin

\section{(2) OpenEdition}

Journals

Édition électronique

URL : http://journals.openedition.org/apliut/6653

DOI : 10.4000/apliut.6653

ISSN : 2119-5242

Éditeur

APLIUT

Ce document vous est offert par Université de Lorraine

UNIVERSITÉ

DE LORRAINE

Référence électronique

Nicolas Molle, Justine Paris et Carine Martin, « D'un service transversal prestataire de services à une UFR décisionnaire de la politique des langues : exemple concret de la structuration d'une UFR Lansad à l'université de Lorraine et de ses impacts en termes de recherche et formations », Recherche et pratiques pédagogiques en langues de spécialité [En ligne], Vol. $38 \mathrm{~N}^{\circ} 1$ | 2019, mis en ligne le 15 mars 2019, consulté le 23 novembre 2019. URL : http://journals.openedition.org/apliut/6653 ; DOI : 10.4000/apliut.6653

Ce document a été généré automatiquement le 23 novembre 2019. 


\section{D'un service transversal prestataire de services à une UFR décisionnaire de la politique des langues : exemple concret de la structuration d'une UFR Lansad à l'université de Lorraine et de ses impacts en termes de recherche et formations}

From a Cross-Department Service Provider to a Policymaker for Languages: The Example of the UFR Lansad at the University of Lorraine and its Impact in Research and Student Training

Nicolas Molle, Justine Paris et Carine Martin

\section{Introduction}

Brudermann et al. soulignent que l'un des problèmes majeurs du secteur des langues pour spécialistes d'autres disciplines (Lansad) réside dans l'absence de lien entre recherche et offre de formation (Brudermann et al. 9, Commission Formations de la SAES 40-65). Les activités de recherche en secteur Lansad sont en effet rares à l'heure où l'Université se doit de proposer une offre de formation qui s'appuie sur la recherche (Brudermann et al. 9). Toutefois, il convient de prendre ici l'exemple de la création d'une UFR Lansad à l'université de Lorraine, fruit des évolutions parallèles de la recherche et de l'offre de formation en langues pour les spécialistes d'autres disciplines, afin de témoigner de l'existence d'un tel lien. 
2 À cette absence de lien entre formation et recherche, Braud et al. ajoutent un second problème, celui de l'absence de vision politique des langues au sein des établissements d'enseignement supérieur :

De nouvelles structures sont créées sans pour autant que ces créations s'appuient sur une politique des langues qui, lorsque le terme est employé, correspond bien trop souvent au « maintien du statu quo ». (Braud et al. 48)

3 En 2006, Petit avait déjà fait ce constat (Petit 3). Néanmoins, l'UFR Lansad de l'université de Lorraine est l'exemple d'une prise de conscience d'une nécessaire politique des langues au sein de l'université. Ainsi, sa création est le point d'orgue d'un cheminement des instances politiques de l'université de Lorraine associé à l'aboutissement de réflexions didactiques menées par les enseignants-chercheurs dans les différentes structures historiques, ancêtres de l'UFR actuel. Nous proposons donc de voir dans quelle mesure la structuration du Lansad au sein d'une université peut avoir un impact sur les formations et sur leur adossement à la recherche.

\section{D'un service transversal à la création d'une UFR Lansad : la nécessaire implication de la recherche dans le processus de reconnaissance politique de l'institution}

\subsection{Une première prise de conscience : l'équipe « Anglais Deug non spécialistes »}

4 En 1973, une équipe de quatre enseignants d'anglais ${ }^{1}$ se constitue, avec pour objectif de fournir un enseignement aux diverses filières de l'Université de Nancy 2 - Droit, Sciences Économiques, Philosophie, Psychologie, Sociologie et Sciences des Communications ${ }^{2}$. Malgré la taille réduite de l'équipe et de la variété des statuts (professeurs certifiés, doctorante et lectrice), dès sa création, ces enseignants étaient en proie à des questionnements didactiques, en particulier sur la notion d'anglais de spécialité (Fade 2). Toutefois, face à l'augmentation des demandes (notamment en termes de diversité des langues) et devant la nécessité d'une coordination plus efficace, l'université de Nancy 2 décide de se doter d'un service transversal commun à tous les départements : le Service commun d'enseignement des langues vivantes (Scelv).

\subsection{D'une structure à une autre : du Scelv au pôle d'enseignements d'autoformation et ressources en langues (Pearl)}

5 En 1991, le Scelv voit donc le jour. À l'instar de l'équipe de 1973, l'objectif demeurait similaire avec, néanmoins, un élargissement de son champ de compétences. En effet, l'anglais n'était plus la seule langue à être enseignée : allemand, espagnol, grec, italien et russe étoffèrent l'offre de formation. Le champ d'intervention de cette structure était également élargi avec des enseignements dispensés à toute la faculté de Droit, Économie et Gestion et à l'Institut supérieur d'administration et de management. À cela, s'ajoutèrent des moyens en termes de fonctionnement alloués par l'université de Nancy 2. Ainsi, la directrice du tout premier service commun, accompagnée par neuf enseignants (sept professeurs agrégés et certifiés - Prag/PRCE, un enseignant-chercheur et un 
lecteur), comptait sur l'appui d'une secrétaire. Sur le plan purement pédagogique, le Scelv bénéficiait de différents outils, tels que la Bibliothèque Sonore-Vidéothèque, devenue le Centre de Langues Yves Châlon (Clyc) en 1996. En 2010, le Scelv comptait une quinzaine d'enseignants titulaires de différents statuts. Toutefois, n'étant pas associée directement à un laboratoire de recherche, la catégorie des Prag et PRCE constituait les principaux enseignants du service, épaulés par trois enseignants-chercheurs. Enfin, en 2010, le Scelv a changé d'acronyme pour devenir le Pôle d'Enseignement Autoformation et Ressources en Langues (Pearl). Ce changement de nom trouve son origine dans l'intégration du Clyc, résultat de la collaboration étroite des enseignants du Scelv et du Crapel. En effet, les différentes activités de recherche menées par les enseignants-chercheurs sur les notions d'autonomie et d'autoformation ont mis en évidence la nécessité de lier les enseignements en présentiel avec un parcours d'autoformation en centres de ressources en langues (CRL) - parcours prenant davantage en compte les besoins personnels des apprenants (Bailly et Carette 77).

\subsection{Les défis de la création d'une UFR Lansad}

6 Le passage de l'université de Nancy 2 à l'université de Lorraine en 2012 mena à une révision structurelle de l'université - le statut administratif d'un service commun n'était alors plus adapté. Le nom du Pearl subit alors une dernière modification afin de remplacer la signification $d u$ « $R$ » de « ressources » par « recherches». En effet, le lien enseignement/recherche n'était plus à démontrer (comme nous le verrons en troisième partie) et, avec la création d'un département CRL à l'intérieur de la future UFR Lansad, il n'était plus pertinent d'associer le terme « ressources » au département. En outre, il y eut une prise de conscience des faiblesses du fonctionnement du Pearl, trouvant un écho dans l'enquête de la SAES en 2015 et dans de nombreux articles (Brudermann et al. 5-8; Braud et al. 51). La première faiblesse était de nature administrative puisque le Pearl fournissait des enseignants à toutes les filières qui inscrivaient des langues obligatoires dans leur maquette. La gestion d'un tel service était devenue une tâche impossible pour une seule directrice et une seule secrétaire avec 19 enseignants titulaires et 30 personnels extérieurs aux multiples statuts (enseignants-chercheurs, Prag, PRCE, vacataires, contractuels et lecteurs). En outre, voué à être un service transversal, la structure était perçue comme un simple prestataire de services devant se plier aux desideratas des responsables de diplômes - qui avaient le réflexe de contacter le département Langues, Littératures et Civilisations Étrangères et Régionales (LLCER) pour la prise en charge des enseignements de leur maquette car, dans leur esprit, ces départements étaient gage de qualité. Il convient aussi de rappeler que le secteur Lansad est relativement récent et jouit donc de peu de reconnaissance de la part de ses homologues spécialistes (Rivens Mompean « Modélisation » 5). Ce manque de considération était sans doute lié au fait que le Scelv, puis le Pearl, renvoyaient à des services exclusivement d'enseignement. Cependant, bien que des recherches aient toujours été menées par les enseignantschercheurs du Pearl au sein du Crapel, pour l'extérieur, il n'y avait pas de lien évident entre enseignements et recherche. Ainsi, ce manque de visibilité contribua sans doute à la perception du Pearl comme étant un simple prestataire de services, bon à fournir la main d'œuvre nécessaire aux enseignements des langues définis par les responsables de diplômes sans concertation avec les spécialistes de l'apprentissage des langues. 
7 Aussi, avec la prise de conscience de l'inadéquation administrative du Pearl, la reconnaissance des recherches menées au Crapel par les instances politiques ${ }^{3}$, ainsi que la reconnaissance de l'apprentissage en CRL, une nouvelle structure devait être créée. Une structure qui serait identifiée et composée de spécialistes en didactique des langues et vue comme force de propositions en politique d'apprentissage des langues étrangères grâce à une expertise solide et fondée sur la recherche. L'UFR Lansad, symbole de cette nouvelle conception, vit donc le jour.

\section{L'UFR Lansad : une structure visible, organisée et fédératrice des diversités}

8 La création de l'UFR Lansad a entraîné des changements structurels importants au sein des anciens services et un véritable changement de paradigme au niveau de la reconnaissance de ce secteur et des rapports avec les autres composantes de l'université de Lorraine - sur lesquels nous revenons en détails ici.

\subsection{Structure et missions de l'UFR Lansad}

9 L'UFR est composée de trois départements :

- le département CRL - Lorraine, qui regroupe trois centres de langues situés sur différents campus,

- le Défle - Lorraine, chargé de coordonner les enseignements de français langue étrangère à Nancy et à Metz,

- Pearl, qui assure les enseignements en allemand, anglais, chinois, espagnol et italien pour les étudiants spécialistes d'autres disciplines.

10 L'équipe administrative de l'UFR compte cinq secrétaires encadrées par une responsable administrative. L'équipe enseignante compte 89 enseignants dont 37 permanents. Le volume d'enseignement a atteint 19000 heures équivalent TD pour l'ensemble de l'UFR Lansad en 2017-2018.

11 L'institutionnalisation du secteur Lansad transparait particulièrement dans certaines des missions de l'UFR définies dans ses statuts :

12 - «Favoriser la mutualisation de l'enseignement des langues et du FLE pour les composantes de l'université de Lorraine». Cette mutualisation s'entend au niveau des enseignements et des apprentissages mis en place, mais également en termes de partage d'outils et de pratiques pédagogiques. Il s'agit d'une institutionnalisation de la dimension fédératrice du Lansad: l'UFR Lansad fédère la diversité des besoins des étudiants des composantes avec lesquelles elle travaille.

13 - « Développer une politique ambitieuse et innovante d'enseignement des langues pour spécialistes d'une autre discipline ». Ce point est essentiel puisque par ce biais, l'université confie la conception et la mise en place de la politique des langues relative à ce public à l'UFR Lansad. Au lieu d'être de simples exécutants d'une politique des langues définie de façon extrinsèque, les membres de l'UFR Lansad deviennent instigateurs de cette politique. Pour preuve, l'UFR a participé à la rédaction du protocole de mise en œuvre de la nouvelle offre de formation 2018 pour ce qui relève de la politique des langues. Ce basculement est rendu possible par l'adossement des formations à la recherche, assurée notamment par la participation des enseignants-chercheurs aux 
travaux du Crapel, comme il le sera démontré dans la partie suivante. La place de la recherche est donc fondamentale au sein de l'UFR. Elle permet de fédérer l'équipe enseignante dans toute sa diversité, ainsi les enseignants-chercheurs de l'UFR ont également vocation à participer à la formation des futurs enseignants de langues appelés à enseigner aux publics Lansad. Des journées de formation internes à l'UFR sont organisées chaque année. À cela s'ajoute l'organisation régulière d'une journée des langues ouvertes aux collègues des autres UFR ayant un public Lansad. Enfin, l'essaimage de la recherche en didactique réalisée au Crapel au sein de l'équipe Autonomie et Apprentissage Autodirigé se fait également sur le terrain avec la mise en place d'équipes pédagogiques mixtes - incluant enseignants et enseignants-chercheurs.

14 - « Construire une offre de services ». L'UFR a vocation à élaborer une offre de formation en langue capable de répondre aux besoins de l'université de Lorraine et du monde socioéconomique. Cette offre reflète la politique des langues de l'UFR, en particulier son approche par compétence et surtout son engagement dans les pratiques pédagogiques innovantes centrées sur l'étudiant et visant à son autonomisation. L'offre de formation du Pearl (voir Annexe 1) met en exergue trois axes fédérateurs au sein du Lansad dans sa globalité. Le premier est la recherche, en particulier dans le domaine de l'autonomisation de l'apprenant et des Tice. L'apprenant est sensibilisé aux démarches d'apprentissage autonomes à travers une formation sur la méthodologie de l'apprentissage en licence et master (que nous détaillons en 3.1.2 et 3.2.2). Le second axe s'incarne dans le CRL qui est le pivot des formations en langues que ce soit au Pearl ou au Défle (sur lequel nous revenons en 3.2.2). Enfin le dernier axe est la certification comme objectif à atteindre pour toutes les langues, le diplôme d'études en langue française (Delf) et le diplôme approfondi de langue française (Dalf) en ce qui concerne le FLE, et les certificats de compétences en langues de l'enseignement supérieur (Cles) pour les autres langues.

La structuration du secteur Lansad par la voie institutionnelle avec la création d'une UFR a donc permis de fédérer trois entités - les CRL, le service d'enseignement du FLE ainsi que le service d'enseignement des langues étrangères - autour d'objectifs et de moyens communs.

\subsection{Effets de la structure sur la reconnaissance des formations Lansad}

La mise en place de l'offre de formation Lansad pour la nouvelle accréditation 2018-2022 met en perspective les effets de la structure sur la reconnaissance du secteur Lansad. La structuration en UFR a permis de changer la nature des relations avec les autres composantes, en passant d'un système bottom-up à un système top-down. Pour préparer l'accréditation précédente (2013-2018), la directrice du Pearl avait dû militer pour la place des langues à l'intérieur des maquettes de chaque département. Ces dernières étaient ensuite transférées aux UFR puis aux collegiums (regroupements de composantes). Cette approche bottom-up était coûteuse en temps et en énergie puisqu'il a fallu convaincre chaque directeur de département. De plus, elle favorisait clairement les freins au changement puisque les décisionnaires étaient les directeurs de département - qui ne sont pas forcément sensibilisés à la question des langues, pris au piège de leurs propres représentations de ce qu'est un enseignement de langue ou qui privilégiaient les heures disciplinaires au détriment des heures de langues. 
17 La création de l'UFR Lansad a, au contraire, suscité une dynamique top-down qui a permis de lever de nombreux freins à l'homogénéisation de l'enseignement des langues. La présidence de l'université de Lorraine a en effet chargé l'UFR Lansad de créer une offre de formation, ce qui a permis à l'UFR d'adopter un positionnement stratégique dans ses relations avec les composantes.

Cette fois-ci, l'offre de formation a été transmise à chaque collegium, charge à chaque entité de l'adopter ou non. Il y a donc eu un changement d'interlocuteurs qui n'étaient plus les directeurs de départements mais les directeurs de collegium (ALL, SHS, DSEG et LMI-Lorraine Management Innovation). Le nombre réduit d'interlocuteurs a facilité le travail de communication nécessaire autour de l'offre de formation - et surtout, la communication s'est faite avec les pouvoirs décisionnaires de chaque composante. Ainsi non seulement les interlocuteurs ont changé, mais les appuis également. Ce travail de communication a été mené par la directrice de l'UFR Lansad, qui a pu compter sur la directrice du collegium dont dépend l'UFR Lansad, ainsi que sur la vice-présidente formation et la vice-présidente de l'université pour relayer l'information au sein des composantes. L'argument de la recherche, de l'innovation pédagogique ainsi que le soutien affiché de la présidence ont permis l'adoption de l'offre de formation par toutes les composantes et la levée de réticences quant à l'approche pédagogique proposée. Cette dynamique top-down a mis l'UFR dans une position de force pour l'accréditation 2018-2022, et pour la première fois, ce sont les spécialistes de l'enseignement des langues qui ont décidé du nombre d'heures maquettées, du contenu des enseignements, des effectifs et des modalités de contrôle des connaissances. De plus, elle a permis à l'UFR de gagner en reconnaissance de par une visibilité accrue auprès des directeurs et directrices de collegium et d'UFR et une systématique mise en avant du caractère innovant des formations Lansad par la vice-présidente et la vice-présidente formation.

La structuration du secteur Lansad par la voie institutionnelle à l'université de Lorraine a donc eu de nombreuses retombées. La conjonction de la volonté politique de l'université de Lorraine et l'expertise scientifique du Crapel a favorisé la création d'une offre de formation prenant en compte la diversité du public Lansad tout en œuvrant à une mise en commun et à une structuration cohérente des objectifs de formations entre les différentes langues. De plus, la mise en place de cette offre de formation a permis à l'UFR de gagner en reconnaissance auprès des diverses composantes de l'université de Lorraine en se positionnant comme expert dans le domaine de l'enseignement des langues vivantes et porteur de la politique des langues de l'université. Nous revenons maintenant plus en détails sur la nature des formations en langue que propose l'UFR.

\section{Les enseignements de langues au sein de l'UFR Lansad}

\subsection{Les travaux du Crapel et leur influence sur les enseignements de l'UFR}

Les enseignements de langues dispensés au sein de l'UFR Lansad sont très empreints des travaux scientifiques du Crapel dans le domaine de l'autonomie et de l'apprentissage autodirigé. Nous revenons sur ce cadre théorique avant d'illustrer la manière dont cet 
éclairage scientifique a influencé les pratiques pédagogiques des enseignants de l'UFR Lansad et leur conception de dispositifs.

\subsubsection{Les concepts scientifiques d'autonomie et d'apprentissage autodirigé en langue}

Henri Holec, pionnier des réflexions et des recherches dans ce domaine, définit l'autonomie d'un apprenant comme sa "capacité de mener, activement et de manière indépendante, un apprentissage » (Holec, «L'apprenant » 11). Apprendre ne se réduit pas à réaliser des "actes d'apprentissage »: c'est aussi être capable de définir ses propres objectifs, contenus et modalités de réalisation, de choisir des ressources et méthodes en conséquences, d'assurer le suivi de ses avancées et de les évaluer (Holec, "Qu'est-ce qu'apprendre? » 80-81). Du point de vue de l'apprentissage des langues, il est donc tout aussi important que l'apprenant développe une certaine autonomie langagière ou linguistique dans la langue cible (aptitude à communiquer dans cette langue) qu'une autonomie d'apprentissage dans la langue cible (savoir apprendre cette langue). Pour Little, autre pionnier dans le domaine de l'apprentissage autodirigé, l'autonomie d'un apprenant renvoie à ses capacités d'apprendre pour lui-même - plus que par lui-même (14). Sa conception s'inspire de la psychologie sociale où l'autonomie est appréhendée comme le fait d'agir librement, avec volonté, et en faisant ses propres choix (Little 17). Il est ainsi important que les apprenants se sentent concernés par ce qu'ils apprennent (les apprentissages formels leur apparaissent souvent comme déconnectés de la réalité), qu'ils puissent se construire leurs propres représentations (plutôt qu'elles ne leurs soient communiquée de manière transmissive), et prendre de la distance avec ce qu'ils font (Little 19-20). Tout comme chez Holec, apprendre une langue pour Little, c'est développer à la fois une autonomie langagière et une autonomie d'apprentissage. Se sentir plus autonomes dans leur apprentissage permettra aux apprenants d'être plus agentifs et, en conséquence, de progresser dans la langue (conférence plénière congrès Cercles 2016). Ce sentiment de contrôle contribue à renforcer l'autonomie générale de l'individu - à savoir le souhait de chacun d'avoir le contrôle de sa propre vie (Benson 16). Il s'agit donc d'un sentiment d'autonomie de premier ordre, qui a toute son importance puisqu'il s'agirait de l'un des trois piliers de la notion de motivation (avec le sentiment de compétence et la reliance aux autres) comme le rappellent Toffoli et Speranza (3).

Le rôle qui est donné à l'enseignant dans le domaine de l'apprentissage autodirigé est alors quelque peu différent de celui qu'il tient habituellement en classe. De détenteur et distributeur de savoirs, supports ou activités pédagogiques, l'enseignant devient un compagnon d'apprentissage. Sa mission est de réunir toutes les conditions pour permettre à l'apprenant de gérer son apprentissage de la langue tout en en lui laissant la responsabilité des actions et des activités qu'il souhaite mener (Little 24). Il s'agit d'un rôle pédagogique qui s'apparente à celui de conseiller : l'enseignant n'est pas là pour prendre les décisions les mieux adaptées pour l'apprenant mais pour apporter une aide à la prise de décision (Gremmo 174, Benson 17) - « un bon père ne donne pas de viande à son fils. A la place, il lui donne un arc et des flèches et lui apprend à chasser $»^{4}$ (Kuchah et Smith 134).

Pour promouvoir l'autonomisation de l'apprenant, la mise à sa disposition de ressources diverses et variées où puiser des supports est donc clé. Pour que l'apprenant soit à même de savoir comment les sélectionner, les utiliser et les combiner afin d'établir un 
programme d'apprentissage, ces ressources doivent faire partie d'un système pédagogique organisé et clair (Gremmo et Riley 97). Le CRL apparait donc souvent comme le lieu de référence aussi bien pour l'apprenant en situation d'autoapprentissage que pour l'enseignant porteur de dispositifs pédagogiques autonomisants (Chateau et al., "Vers l'institutionnalisation » 3 ; Chateau et Bailly, «Autonomie » 65). De plus, un CRL propose souvent des services permettant de pratiquer les langues et d'interagir entre pairs en complément de son offre de ressources - ateliers, conversations avec des natifs, entretiens-conseils (Gremmo et Riley 95), propositions de tandems (Chateau et Bailly, « Learning " 373 ; Bailly et al. 2-3), soirées projections de films, chats, quizz culturels, évènements au moment de commémorations nationales, dégustations, soirées jeux et divers clubs - d'après les communications présentées au congrès annuel du rassemblement national des centres de langues de l'enseignement supérieur (Ranacles) en 2017. Ces services sont la véritable plus-value du CRL qui font de cet espace le lieu privilégié des pratiques informelles (Rivens Mompean, webinaire à la troisième minute).

Tout ceci ne peut cependant fonctionner que si l'apprenant adopte une nouvelle posture. Il doit en effet accepter de s'éloigner de l'environnement d'apprentissage traditionnel où l'enseignant mène la danse. D'un être passif qui se contente de suivre aveuglément le programme pédagogique de son enseignant et de se plier à ses demandes, l'apprenant devient meneur à son tour et élabore lui-même son propre plan d'action (Holec, «Qu'estce qu'apprendre?» 76; Gremmo 174). Aussi difficile que cela puisse paraitre, les apprenants en sont capables - Bailly et al. ont même montré que les apprenants appréciaient souvent ce recentrage sur soi et la dimension «sur-mesure " que l'apprentissage autodirigé leur offre (13). De nombreuses expérimentations pédagogiques dans les enseignements de l'UFR Lansad ont également montré que des dispositifs pédagogiques autonomisants peuvent être mis en place à grande échelle, comme nous allons le voir maintenant.

\subsubsection{L'application directe de ce paradigme dans les enseignements dispensés au sein de I'UFR Lansad}

Le cadre théorique des recherches dans le domaine de l'apprentissage autodirigé se retrouve dans de nombreux enseignements de l'UFR Lansad - qui cherchent à promouvoir l'autonomisation de ses étudiants. Le cours d'anglais pour les étudiants en M1 de Psychologie, qui compte chaque année environ 180 étudiants, en est l'exemple le plus révélateur (Chateau et Zumbihl, «Le carnet de bord » 2-4; Chateau et Zumbihl, « From the logbook » 2 ; Chateau et Candas 399-400).

Dans sa forme actuelle, le cours s'articule autour de quatre tâches que les étudiants ont à réaliser librement au cours du semestre. Il s'agit d'un enregistrement-audio pour travailler la prononciation en profondeur, d'un résumé-commentaire sur un document vidéo en psychologie pour travailler la langue de spécialité, de trois activités interactives semblables aux Tests sous Moodle pour une pratique de la langue générale (Chateau 2-5 les décrit en détails) et d'un carnet de bord à rédiger tout au long du semestre aussi bien pour consigner le travail accompli et assurer le suivi de l'étudiant que pour développer sa capacité de réflexion sur son expérience d'apprenant. Du point de vue du planning du semestre, chaque étudiant se voit proposer cinq séances en présentiel sur les 12 semaines du semestre. Trois séances thématiques sont proposées avec deux ou trois dates au choix afin de leur permettre d'organiser leur semestre comme ils le souhaitent. La toute première séance du cours s'attache à poser un cadre de départ pour le format du cours et 
pour les trois séances thématiques en encourageant les étudiants à s'interroger sur leurs représentations de l'anglais et faire un point sur leurs expériences personnelles avec cette langue. La dernière séance vient alors clore le cours en leur permettant de compléter les tâches inachevées en présence des enseignantes qui viennent à leur rencontre. Trois autres séances (une toutes les quatre semaines) sont consacrées à l'annotation des carnets de bord de chacun des étudiants par les enseignantes afin de les encourager de manière régulière et de leur apporter soutien et conseils en cas de difficulté ou désarroi. Pour chaque tâche du cours, les étudiants ont le choix entre différentes ressources afin de personnaliser leur expérience d'apprentissage au maximum et de les responsabiliser. Et à chaque nouvelle étape du cours, ils sont suivis individuellement ou en groupe par les enseignantes via les carnets de bord ou les temps de présentiel. Le dispositif est donc conçu pour donner aux étudiants une grande marge de manœuvre dans la façon dont ils souhaitent s'organiser pour le cours et dans les ressources qu'ils souhaitent utiliser, tout en assurant un guidage et une collaboration enseignant-étudiants et étudiants-étudiants régulière.

Ce cours a fait l'objet de nombreuses recherches-actions depuis sa création en 2005-2006 sur la base d'analyses quantitatives et qualitatives de carnets de bord et de questionnaires d'évaluation du cours par les étudiants. Elles ont montré que les étudiants avaient une attitude généralement positive sur le déroulé du cours, même s'ils sont souvent réticents et anxieux en début de semestre (Chateau et Zumbihl «From the logbook» 3-4). Elles ont également permis de constater une grande évolution en termes d'autonomie d'apprentissage chez les étudiants («Le carnet de bord» 6-10 - même si certains n'ont pas du tout adhéré au dispositif). Par exemple, $70 \%$ des carnets de bords recueillis en 2012-2013 faisaient état d'événements, situations et activités menant à une prise de conscience sur l'expérience d'apprentissage de la part de leur auteur (Chateau et Candas 402). Le dispositif semble donc avoir facilité le processus d'autonomisation des étudiants grâce à un fort accent sur la réflexion personnelle au sujet de ses propres pratiques.

Depuis quelques années, d'autres dispositifs autonomisants ont fait leur apparition dans les enseignements de l'UFR Lansad avec la particularité de s'appuyer sur les services offerts par les CRL de l'université. Avant de s'intéresser à ces dispositifs, nous revenons brièvement sur l'environnement d'apprentissage qu'ils proposent.

\subsection{Le centre de ressources en langues : pivot des enseignements de l'UFR Lansad}

\subsubsection{Le fonctionnement des centres de ressources en langues de l'UFR Lansad}

29 A l'occasion de l'élaboration de la nouvelle offre de formation en 2013, il a été décidé que six heures équivalent TD de chacun des cours de langues relevant du Pearl seraient reversées au département CRL de l'UFR Lansad afin de pouvoir rémunérer ses intervenants. Car outre l'accès à de nombreuses ressources physiques et numériques (méthodes de langues, dictionnaires, DVD, bandes-dessinées, sites et applications d'apprentissage gratuits et payants, etc.), les CRL de l'université de Lorraine proposent trois types de services pédagogiques - les ateliers, les conversations et les entretiensconseils. Les ateliers sont des séances thématiques, très souvent culturelles, qui proposent diverses activités aux apprenants. Par exemple, un atelier en anglais sur la folie Star Wars propose aux étudiants de faire un test de personnalité pour savoir à quel personnage de la saga ils ressemblent le plus, tester leurs connaissances de la saga via un 
jeu de cartes où ils doivent assembler les titres des films et leur année de parution, lire un article sur les différents coups marketing du film et en discuter en groupe. Les conversations, avec des locuteurs natifs, ont pour but de faire pratiquer l'expression orale de manière libre et le plus spontanément possible. À l'instar des ateliers, elles sont limitées à huit participants afin de donner l'opportunité à chacun de s'exprimer dans un climat détendu. Enfin, les entretien-conseils offrent la possibilité aux étudiants de prendre rendez-vous individuellement avec un conseiller en apprentissage des langues étrangères pour faire le point, demander de l'aide, ou avoir un suivi sur son apprentissage d'une langue.

De façon à proposer un environnement d'apprentissage optimisé, un projet subventionné en 2015 par la région Lorraine et le fonds européen de développement régional (FEDER) a permis la réalisation d'une plateforme numérique ${ }^{5}$ fédératrice des trois CRL de l'université (Chateau et al. «Constribution » 3-5). Véritable interface entre les CRL, leurs intervenants et les étudiants, cette plateforme permet de centraliser les informations pratiques sur les différents CRL (adresses, horaires, etc.) et facilite la gestion des services (les intervenants pédagogiques peuvent y publier leurs annonces d'ateliers ou de conversations et les étudiants peuvent s'y inscrire en différé). La plateforme compte également un accès à un catalogue qui répertorie les ressources des trois CRL que même qu'à des tutoriels sur l'apprentissage des langues ${ }^{6}$ et un carnet de bord interactif personnel $^{7}$ (qui consigne le temps passé sur la plateforme ou en CRL, les ressources consultées et les services auxquels ils ont participé). Enfin, la plateforme dispose d'une rubrique sur l'apprentissage en autodirection à destination aussi bien des étudiants que des intervenants pédagogiques et des chercheurs qui souhaiteraient davantage d'informations dans ce domaine ${ }^{8}$.

31 C'est donc un environnement d'apprentissage qui s'efforce d'être le plus diversifié et complet possible qui est proposé aux étudiants - combinant à la fois un espace physique et un espace numérique, des ressources aux formats et accès variés, et des services de pratiques informelles des langues. La plateforme EDOlang, et l'hybridité des pratiques qu'elle a permis de développer, a eu une grande influence sur les pratiques enseignantes à compter de sa mise en ligne en 2015. Les enseignants de l'UFR Lansad sont plus nombreux chaque année à intervenir dans les CRL dans le cadre d'ateliers et d'entretiens-conseils de même qu'à introduire le CRL dans leur plan de cours comme nous allons le voir maintenant (Chateau et al. 7-8).

\subsubsection{L'institutionnalisation des CRL à l'UFR Lansad}

32 Même si le CRL se veut le lieu de l'apprentissage autodirigé - un espace, certes institutionnel, mais indépendant des formations diplômantes des étudiants - les CRL sont de plus en plus utilisés en complémentarité des enseignements dispensés au sein de l'UFR Lansad.

3з C'est le cas notamment de la formation en anglais au niveau licence pour toutes les filières d'Art, Lettres, Langues et SHS à Nancy. Au premier semestre de la L1 de toutes ces filières, les étudiants ont un rallye découverte en autonomie à réaliser au CRL du campus Lettres et Sciences Humaines et Sociales, et des questions sur ce rallye leur sont posées au partiel de fin de semestre. Au premier semestre de la L2, deux séances sont prévues en CRL où les étudiants doivent travailler sur des documents sonores de leur choix issus de sites d'apprentissage proposés au CRL. Ils sont encadrés par les enseignants qui passent 
les rencontrer un à un pour les assister dans leur travail personnel de compréhension de l'oral. Au semestre suivant, une séance a lieu en CRL dans le cadre d'une séance consacrée à l'expression écrite. Les étudiants ont alors le choix entre une sélection de méthodes de langues papier et interactives et ils doivent décider des points de langue sur lesquels ils souhaitent travailler en vue de la préparation d'un travail sur une conférence TEDx. Les enseignants apportent suivi et conseils à chaque étudiant pendant la séance. Enfin, au premier semestre de la L3, les étudiants doivent participer à une conversation ou à un atelier en CRL en complément de séances consacrées à l'expression orale.

Au niveau master, le CRL est régulièrement utilisé pour personnaliser les parcours de formation des étudiants. C'est le cas du cours d'anglais de M1 Sciences de l'Education : au deuxième semestre, les étudiants ont le choix entre se présenter à certaines séances du cours ou aller travailler librement en CRL. Cette population d'étudiants travaillant généralement sur trois types de sujets dans le cadre de leur mémoire de M1 - l'enfant, l'adolescent et l'adulte (pour un travail personnel sur l'enseignement au niveau primaire, secondaire, supérieur ou en formation continue), il ne paraissait pas pertinent à l'enseignante en charge de cet enseignement d'imposer un travail fin sur le vocabulaire de chacun de ces sujets à tous les étudiants. De plus, ces séances présentent toute la même structure : un travail de compréhension écrite sur un article scientifique via une activité Test sous Moodle à réaliser avant de se présenter au cours, un travail sur le vocabulaire transversal des études sur l'enfant / l'adolescent / l'adulte en Sciences de l'Education en anglais en classe, et une étude de cas. Assister à chacune de ces trois séances aurait été à la fois chronophage pour tous les étudiants et peu utile pour ceux dont le thème de recherche ne renvoyait qu'à un seul type de ces sujets. Ils ont donc pu choisir de ne pas se présenter à certaines de ces séances et passer autant de temps en CRL à travailler leur anglais en autodirection selon leurs besoins langagiers personnels. Après chaque séance de travail autodirigé en CRL, les étudiants devaient rédiger un paragraphe analytique sur leur expérience d'apprenant durant cette séance - à savoir un retour ce qu'ils avaient aimé / moins aimé, trouvé facile / difficile, les possibles raisons de leurs difficultés et les moyens qu'ils ont alors mis en place pour contourner leur difficulté ou envisagés pour s'améliorer suite à cette séance. Lors du premier semestre, les étudiants avaient dû rédiger un carnet de bord tout comme les étudiants de M1 Psychologie, ils avaient donc déjà eu l'occasion de réfléchir et s'exprimer sur ces questions.

On observe donc une grande complémentarité entre la classe de langue et le CRL au sein des enseignements dispensés à l'UFR Lansad qui s'attache à créer une synergie entre la classe de langue et le CRL dans la lignée d'autres UFR de l'université de Lorraine. De plus, les CRL sont très présents dans l'esprit des enseignants de l'UFR : lorsqu'ils ne peuvent pas proposer des activités en CRL en présentiel ou à distance dans le cadre de leurs enseignements, ils n'hésitent pas à rappeler régulièrement les opportunités de travail personnel qu'offrent les CRL à leurs étudiants. Ils projettent également régulièrement la plateforme EDOlang en classe afin de leur remémorer tout ce qu'ils peuvent y trouver, de même que la procédure pour s'inscrire à un service ou consulter une ressource. Depuis la création de cette plateforme en 2014, la fréquentation des CRL ne cesse d'augmenter : en 2011-2012, 46 inscriptions à des entretiens-conseils ont été comptabilisées contre 81 en 2015-2016; 888 inscriptions en conversations en 2011-2012 contre 1482 en 2015-2016 (Château et al 6). 


\section{Conclusion}

l'université de Lorraine laisse apparaître le rôle clé de la recherche dans la reconnaissance institutionnelle de ce secteur. Un nombre grandissant de Maîtres de Conférences et leur implication dans les domaines de l'autonomisation et des CRL a permis de remodeler le contenu et la structuration des enseignements de toute l'équipe enseignante. Ceci a engendré une prise de conscience au niveau de l'institution et la création d'une UFR Lansad.

our fédérer le secteur Lansad, la piste institutionnelle semble prometteuse : une UFR rassemble les enseignants dans une même structure, clairement identifiée au sein de l'université. Elle favorise la mise en place d'une politique des langues avec une structuration cohérente des objectifs de formation en lien avec la recherche. Elle permet également de gagner en considération auprès des autres composantes de l'université avec lesquelles il devient possible de dialoguer en assurant une position d'expertise.

Des freins sont toujours présents car changer de positionnement implique des remaniements que les UFR partenaires peinent parfois à comprendre mais avec le temps, ces difficultés s'estompent. De plus, il peut exister un écart entre la politique des langues inscrite dans les documents officiels et sa réelle mise en œuvre.

À l'heure où les universités françaises se regroupent en grands établissements, elles ont de plus en plus besoin d'un interlocuteur unique qui puisse prendre en charge et homogénéiser les cours de langues dans l'ensemble de leur structure. Par ailleurs, l'innovation pédagogique est de plus en plus reconnue et mise en avant par l'institution même. Or, comme en témoignent les enseignements proposés au sein de l'UFR Lansad de l'université de Lorraine, le secteur Lansad s'avère très innovant sur le plan pédagogique. Il y aurait donc là un levier à actionner pour plus de reconnaissance institutionnelle du secteur en associant recherche et formation.

\section{BIBLIOGRAPHIE}

Bailly, Sophie, et al. « Reliance et confiance dans un environnement d'apprentissage autodirigé en langues. » Recherche et pratiques pédagogiques en langues de spécialité. Cahiers de l'Apliut, vol. 37 n 1, 2018. journals.openedition.org, doi:10.4000/apliut.5882.

Bailly, Sophie, et Emmanuelle Carette. «Introducing Self-Directed Learning in an InnovationFriendly Institutional Context. » Porta Linguarum, n 6, 2006, pp. 77-97.

Benson, Phill. « What's New in Autonomy? » JALT2011, 2011, http://jalt-publications.org/files/ pdf-article/plen3.pdf.

Braud, Valérie, et al. « You say you want a revolution... » Contribution à la réflexion pour une politique des langues adaptée au secteur Lansad. » Recherche et pratiques pédagogiques en langues de spécialité. Cahiers de l'Apliut, vol. 34, n 1, 2015, pp. 46-66.

Recherche et pratiques pédagogiques en langues de spécialité, Vol. 38 Nㅜ1 | 2019 
Brudermann, Cédric, et al. «Le secteur des langues pour spécialistes d'autres disciplines dans les universités françaises : résultats d'une enquête nationale menée par la SAES. » Recherche et pratiques pédagogiques en langues de spécialité. Cahiers de l'Apliut, $\mathrm{n}^{\circ}$ Vol. $35 \mathrm{~N}^{\circ}$ spécial 1, 2016. journals-openedition-org.bases-doc.univ-lorraine.fr, http://journals.openedition.org.bases-doc.univlorraine.fr/apliut/5564.

Chateau, Anne, et al. « Contribution/soutien des espaces à l'autodirection : un exemple d'hybridation du virtuel et du physique à l'université de Lorraine. » Alsic. Apprentissage des Langues et Systèmes d'Information et de Communication, vol. 20, $\mathrm{n}^{\circ} 3$, 2017. journals.openedition.org, http://journals.openedition.org/alsic/3143.

Chateau, Anne. « Langues-U: A digital Campus to Increase Student's Autonomy? » Language Centres at Universities: Crossing Bridges, Integrating Cultures: papers from the 9th Cercles Conference Frankfurt (Oder), 28-30 September 2006, Frankfurt (Oder) : CERCLES, 2008., Thomas Vogel, Ray Satchell, Alžbeta Moražikova, 2008, pp. 115-22.

Chateau, Anne, et al. «Vers l'institutionnalisation de l'enseignement autonomisant - La technologie, soutien et obstacle à l'innovation. » Alsic. Apprentissage des Langues et Systèmes d'Information et de Communication, vol. 18, n², 2015. alsic.revues.org, https://alsic.revues.org/2838.

Chateau, Anne, et Sophie Bailly. « Autonomie d'apprentissage de langues en CRL : voie unique ou chemin multiples? ? Les langues modernes, $\mathrm{n}^{\circ} 3$, 2012, pp. 62-68.

Chateau, Anne, et Peggy Candas. « Tracking Students' Autonomization through Emotion Traces in Logbooks. » Studies in Second Language Learning and Teaching, vol. 5, n 3, 2015, pp. 395-408. pressto.amu.edu.pl, doi:10.14746/ssllt.2015.5.3.3.

Chateau, Anne, et Hélène Zumbihl. « From the Logbook to the Forum: how to Reinforce Collaborative Learning for a Better Student's Autonomy? » New Trends in Computer-Assisted Language Learning: Working Together. Madrid : Macmillan ELT, 2011, pp. 69-74.

---. « Le carnet de bord, un outil permettant le cheminement vers l'autonomisation dans un dispositif d'apprentissage de l'anglais en ligne? » Alsic. Apprentissage des Langues et Systèmes d'Information et de Communication, vol. 13, 2010. journals.openedition.org, doi:10.4000/alsic.1392.

Commission Formations de la SAES. Livre blanc de la formation en études anglophones. Claire Tardieu et Emily Eells, 2018, http://saesfrance.org/mars-2018-publication-du-livre-blanc-de-laformation-en-etudes-anglophones/.

Fade, Pascale. « L'anglais de spécialité chez les non-spécialistes niveau DEUG. » ASp. la revue du GERAS, nº 1, 1993, pp. 287-300.

Gremmo, Marie-José. « Conseiller en langues : proposition d'analyse de deux décennies de théorie et de pratique(s) pour une approche comparée du tutorat en FOAD ». Dispositifs médiatisés et accompagnement-tutorat, édité par Annick Rivens Mompean et Marie-José Barbot, 2009, pp. 173-90, http://halshs.archives-ouvertes.fr/docs/00/61/98/19/PDF/

Lille_2008_Gremmo_Conseiller_en_langues.pdf.

Gremmo, Marie-José, et Philip Riley. Autonomie et apprentissage autodirigé : l'histoire d'une idée, Site du laboratoire ATILF. 1997, http://www.atilf.fr/spip.php?article3484.

Holec, Henri. « L'apprenant autonome : quelques repères conceptuels. » Babylonia, revue pour l'enseignement et l'apprentissage des langues, $\mathrm{n}^{\circ} 2,1994$, pp. 10-14.

---. « Qu'est-ce qu'apprendre à apprendre ?» Mélanges Pédagogiques, 1990, pp. 75-87. 
Kuchah, Kuchah, et Richard Smith. «Pedagogy of Autonomy for Difficult Circumstances: from Practice to Principles. » Innovation in Language Learning and Teaching, vol. 5, n 2 , 2011, pp. 119-40. Taylor and Francis+NEJM, doi:10.1080/17501229.2011.577529.

Little, David. « Language Learner Autonomy: Some Fundamental Considerations Revisited. » Innovation in Language Learning and Teaching, vol. 1, $\mathrm{n}^{\circ} 1,2007$, pp. 14-29.

Petit, Michel. « Le Mot du Président. » ASp. la revue du GERAS, nº 49-50, 2006, pp. 1-4.

Rivens Mompean, Annick. « Le Centre de Ressources en Langues : missions d'un espace d'apprentissage en évolution. » Alsic. Apprentissage des Langues et Systèmes d'Information et de Communication, 2018. journals.openedition.org, http://journals.openedition.org/alsic/3055.

---. « Modélisation systémique du secteur Lansad : pour une approche durable. » Recherche et pratiques pédagogiques en langues de spécialité, vol. 35, n 2, 2016, http://apliut.revues.org/5410.

Rivens Mompean, Annick et Martine Eisenbeis. « Autoformation en langues : quel guidage pour l'autonomisation?" Les Cahiers de l'Acedle, vol. 6, n 1, 2009, p. 221-244. http://acedle.org/IMG/ pdf/Rivens_Eisenbeis_Cahiers-Acedle_6-1.pdf

Toffoli, Denyze, et Lauren Speranza. « L'autonomie comme facteur déterminant dans la réussite d'un enseignement Lansad en sciences historiques. " Recherche et pratiques pédagogiques en langues de spécialité. Cahiers de l'Apliut, vol. 35 nº spécial 1, 2016. journals.openedition.org, doi:10.4000/ apliut.5505.

\section{ANNEXES}

\section{Annexe - Offre de Formation en Lansad pour Licences}

Objectif en fin de Licence : être prêt à passer une certification en langues dans les 5 compétences correspondant au niveau B2 du CECRL.

Proposition : Modules de formation répartis en heures de présentiel (TD) et heures d' apprentissage autodirigé à l'aide des outils développés par l'UFR Lansad depuis la plateforme EDOlang et des services pédagogiques offerts par l'UFR dans les CRL.

\begin{tabular}{|l|l|}
\hline Semestre 1 & $\begin{array}{l}\text { Semestre } 2 \\
18 \mathrm{hTD}: 12 \mathrm{~h} \text { en présentiel et } 6 \mathrm{~h} \text { d'activités en } \\
12 \mathrm{hTD}: 6 \mathrm{~h} \text { en présentiel et } 6 \mathrm{~h} \text { d'activités en } \\
\text { CRL }\end{array}$ \\
$\begin{array}{l}\text { Axé sur 'apprendre à apprendre en LV' et } \\
\text { autonomisation } \\
\text { Groupes de taille maximale de } 35\end{array}$ & $\begin{array}{l}\text { Axé sur acquisition compétences de } \\
\text { compréhension écrite de textes authentiques en } \\
\text { rapport avec spécialité au sens large (contenu } \\
\text { textes à adapter suivant grands domaines : ALL, } \\
\text { SHS, DEG ...) }\end{array}$ \\
\hline
\end{tabular}




\begin{tabular}{|c|c|}
\hline $\begin{array}{l}\text { Semestre } 3 \\
24 \text { hTD : } 18 \text { h en présentiel et d'6h activités en } \\
\text { CRL } \\
\text { Axé sur acquisition compétences de } \\
\text { compréhension orale de documents } \\
\text { authentiques audio et /ou vidéo en rapport } \\
\text { avec spécialité au sens large (contenu à } \\
\text { adapter suivant grands domaines : ALL, SHS, } \\
\text { DEG ...) }\end{array}$ & $\begin{array}{l}\text { Semestre } 4 \\
24 \text { hTD : } 18 \mathrm{~h} \text { en présentiel et } 6 \mathrm{~h} \text { d'activités en } \\
\text { CRL } \\
\text { Axé sur acquisition compétences d'expression } \\
\text { écrite à partir de documents/ thématiques } \\
\text { authentiques en rapport avec spécialité au sens } \\
\text { large (contenu à adapter suivant grands } \\
\text { domaines : ALL, SHS, DEG ...) }\end{array}$ \\
\hline $\begin{array}{l}\text { Semestre } 5 \\
24 \text { hTD : } 18 \text { h en présentiel et } 6 \text { h de suivi } \\
\text { Axé sur acquisition compétences } \\
\text { d'expression orale à partir de documents/ } \\
\text { thématiques authentiques en rapport avec } \\
\text { spécialité au sens large (contenu à adapter } \\
\text { suivant grands domaines: ALL, SHS, DEG ...) } \\
\text { Groupes de } 25 \text { étudiants maximum pour } \\
\text { l'expression orale }\end{array}$ & $\begin{array}{l}\text { Semestre } 6 \\
24 \text { hTD : } 18 \mathrm{~h} \text { en présentiel et } 6 \mathrm{~h} \text { de suivi } \\
\text { Travail collaboratif sur dossier des } 5 \\
\text { compétences définies dans le CECR, à partir de } \\
\text { documents/ thématiques authentiques en } \\
\text { rapport avec spécialité au sens large (contenu à } \\
\text { adapter suivant grands domaines: ALL, SHS, } \\
\text { DEG ...) }\end{array}$ \\
\hline
\end{tabular}

\section{NOTES}

1. Cette première équipe sous le nom de "Deug Anglais non spécialistes» dépendait du laboratoire de recherche du Centre de recherches et d'applications pédagogiques en langues (Crapel - http://www.atilf.fr/spip.php?article811). Cependant, il est à noter qu'aucun des membres n'était enseignant-chercheur.

2. L'université de Lorraine sera créée en 2012 à la suite d'un processus de fusion d'établissements d'enseignement supérieur en région Lorraine

3. Il faut insister sur le rôle du vice-président du conseil de la formation de l'université qui a facilité la mise en place de la structure.

4. Notre proposition de traduction pour « a good father does not give his son meat. Instead, he gives him a bow and arrow, and teaches him to hunt ».

5. www.edolang.univ-lorraine.fr

6. Ces tutoriels sont disponibles ici: http://edolang.univ-lorraine.fr/fr/construire-monapprentissage/tutoriels-soutien

7. En partie inspiré de Calao, application développée par Lille 3 (Rivens et Eisenbeis ; Chateau et al.).

8. http://coalea.univ-lorraine.fr/accueil 


\section{RÉSUMÉS}

Comme de nombreux articles le soulignent, il n'y pas de politique des langues réellement concertée dans le secteur des Langues pour spécialistes d'autres disciplines (Lansad, Braud et al. ; Brudermann et al. ; Commission Formations de la SAES ; Petit). Ce secteur revêt une multitude de formes et de structures disparates au sein des établissements français, entraînant un manque de reconnaissance institutionnelle. Depuis 2014, l'université de Lorraine s'attache à y remédier à travers son unité de formation et de recherche Lansad (UFR Lansad) - fruit d'une expertise didactique qui a su convaincre les instances politiques de l'université. Cette UFR est aujourd'hui un décideur majeur de la politique de l'enseignement des langues de son université. Cet article s'attache à montrer comment ce modèle a créé un essaimage de formations innovantes inspirées par les travaux de l'équipe de recherche Didactique des Langues et Sociolinguistique (Crapel) et comment sa reconnaissance témoigne de la nécessité de favoriser un lien fort entre recherche et formations.

As many articles point out, there is no clear language policy governing the sector of languages for the specialists of other disciplines (Lansad, Braud et al.; Brudermann et al.; Commission Formations de la SAES; Petit. The Lansad sector takes on many disparate forms and structures within French institutions, leading to a lack of institutional recognition for this field. Since 2014, the University of Lorraine has been working to remedy this situation through its Lansad UFR resulting from a teaching expertise that has convinced the university's political authorities. This UFR is now a major decision-maker in the language teaching policy of the University of Lorraine. This article aims to show how this model has created a spin-off of innovative training inspired by the work of the research team Didactique des Langues et Sociolinguistique (Crapel) and how its recognition demonstrates the need to foster a strong link between research and training.

\section{INDEX}

Keywords : language policy, Lansad sector, self-directed language learning environment, selfaccess learning centers, blended learning, teaching innovation

Mots-clés : politique des langues, secteur Lansad, autonomisation, centres de ressources en langues (CRL), enseignement hybride, innovation pédagogique

\section{AUTEURS}

\section{NICOLAS MOLLE}

Nicolas Molle est maître de conférences en anglais à l'UFR Lansad, directeur du PEARL (Pôle d'enseignement autoformation et recherche en Langues) et membre de l'axe « Autonomie et apprentissage autodirigé » de l'équipe de recherche didactique des langues et sociolinguistique (Crapel) de l'ATILF. Il s'intéresse à l'apprentissage des langues en auto-direction, à la question de l'institutionnalisation du secteur Lansad dans les établissements d'enseignement supérieur ainsi 
qu'à l'anglais comme langue de spécialité.

nicolas.molle@univ-lorraine.fr

\section{JUSTINE PARIS}

Justine Paris est maître de conférences en linguistique anglaise et didactique des langues à l'université de Lorraine. Ses recherches portent sur le langage figuré en acquisition du langage L1/L2 ainsi que sur l'autonomisation de l'étudiant et l'usage de la réalité virtuelle dans le domaine de l'apprentissage des langues. Correspondante « Pédagogie et numérique » pour l'UFR Lansad, elle s'intéresse également à la transformation des pratiques pédagogiques via le numérique et à l'usage des TICE en classe de langue, et plus particulièrement à la pédagogie hybride dans le cadre de l'enseignement de langue de spécialité. Directrice adjointe de l'UFR Lansad en charge de la gestion et promotion des centres de ressources en langues (CRL), elle s'intéresse également à l'apprentissage des langues en auto-direction et aux interactions entre le CRL et la classe de langue.

justine.paris@univ-lorraine.fr

\section{CARINE MARTIN}

Carine Martin est maître de conférences à l'université de Lorraine. Membre de l'équipe CRAPEL de l'ATILF (UMR 7118), elle participe au groupe « Autonomie et apprentissage autodirigé ». Ses recherches portent sur l'autonomisation de l'apprenant, les dispositifs hybrides et plus globalement sur l'intégration du centre de ressources en langues dans l'offre de formation. carine.martin@univ-lorraine.fr 